\title{
Spin-wave control using dark modes in chiral magnonic resonators
}

\author{
K. G. Fripp, A. V. Shytov, and V. V. Kruglyak ${ }^{*}$ \\ University of Exeter, Stocker Road, Exeter, EX4 4QL, United Kingdom
}

(Received 23 April 2021; revised 23 July 2021; accepted 10 August 2021; published 26 August 2021)

\begin{abstract}
We modeled, both analytically and numerically, the magnitude and phase of spin waves propagating in thin magnetic films and scattered from mesoscale chiral magnonic resonators. Our calculations reveal a remarkably strong chiral scattering of propagating spin waves from magnon dark modes hosted by the resonator, exceeding in strength the scattering from its quasiuniform mode. We formulate conditions for the waveguide-resonator system to be used as an efficient spin-wave diode and as a phase shifter. Both these applications are found to be feasible when using the available ferromagnetic materials for the resonators.
\end{abstract}

DOI: 10.1103/PhysRevB.104.054437

\section{INTRODUCTION}

The resonant scattering of spin waves-propagating precessional excitations of the magnetic order [1] - may prove essential for miniaturization of data and signal processing devices of magnonics [2,3]. In general, the resonant scattering's strength is determined by the coupling between the incident waves and the discrete modes of the scatterer. The effect of the coupling is maximized when the frequencies of the incident and discrete modes match. Resonant scattering emerges, e.g., in the theory of spin waves in monomode magnonic waveguides with dangling branches [4] and in two-dimensional arrays of magnetic dots [5], as well as in the theory of spin-wave induced motion of magnetic domain walls $[6,7]$. Experimentally, similar ideas were exploited for spin-wave control in one-dimensional arrays of magnetic stripes [8] and dots [9], as well as in continuous magnonic microconduits [10].

The most important practically and fascinating fundamentally aspect of the resonant scattering of spin waves is its chirality or "handedness," whereby the scattering is nonreciprocal. Although chirality is inherent to magnetism and magnetic phenomena, only recently have its manifestations in magnonics started to be actively explored and exploited. In Refs. [11,12], Au et al. used micromagnetic simulations to demonstrate chirality of the coupling between spin waves propagating in a stripe magnonic waveguide and precessional modes of a nanomagnet placed nearby. The scattering enabled by this chiral coupling was shown to lead to a robust nonreciprocal modulation of the spin-wave amplitude and phase, controlled by flipping the direction of the nanomagnet's magnetization [11]. The spin-wave frequency in Ref. [11] was equal to the value at which the same system acted optimally as a chiral microwave to spin wave transducer [12], i.e., a device concentrating the power of incident microwaves [13] and converting it into unidirectionally propagating spin waves [14]. The scattering in Ref. [11]

\footnotetext{
*v.v.kruglyak@exeter.ac.uk
}

was presumed to be resonant, and its strength and character were shown to depend nonmonotonically both on the damping coefficient in the nanomagnetic resonator and on its spacing from the waveguide. The nonreciprocity of the spin-wave modulation [11] and transduction [12] functionalities was attributed to the chirality of the magnetic precession and associated dynamic stray magnetic field, leading to the magnetization-momentum locking [11].

Here, we use micromagnetic simulations and a phenomenological model to elucidate the resonant character of the chiral scattering of spin waves propagating in thin magnetic films from mesoscale magnonic resonators placed nearby [Fig. 1(a)]. We use the simulated data to calculate both the magnitude and phase of the magnonic transmission and reflection coefficients as a function of the spin-wave frequency and incidence direction and the film-resonator spacing. The transmission is efficiently modulated only for one direction of spin-wave incidence, and this direction is switchable by switching the magnetization of the nanomagnet. Our simulations reveal a remarkably strong chiral scattering of propagating spin waves from magnon dark modes hosted by the resonator, exceeding the scattering from the quasiuniform mode in both strength and chirality. The results of the simulations are interpreted with the help of the phenomenological model, in which the coupling between the spin wave and the resonator is characterized by two contributions to the resonator's radiative linewidths, for the forward and backward emission. In particular, the model predicts the drop in transmission when the forward linewidth is equal to the sum of the backward and dissipative contributions. The transmitted wave's phase shift of $\pi$ at resonance is shown to be a signature of an even stronger and more chiral coupling (when the forward radiative linewidth exceeds the sum of the dissipative and backward radiative linewidths). The scattering line shapes obtained from the simulations are rather symmetric, and the reflection is weak and reciprocal. The model shows that these features are consistent with the chirality of the scattering and the weakness of nonresonant contributions to the scattering. Our simulations are run for spin waves propagating along the direction of the magnetization of the thin film, but the model 

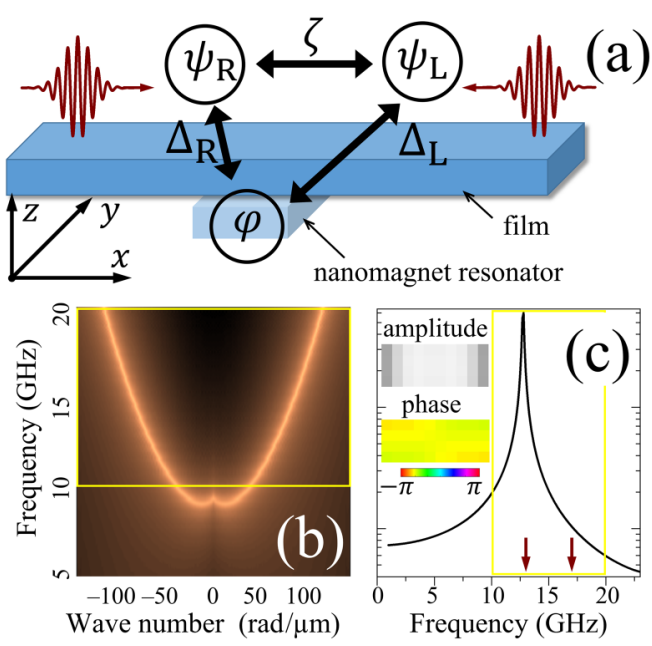

FIG. 1. (a) The geometry of the problem is shown schematically. The nanomagnet plays the role of a chiral nanomagnet resonator (mode $\varphi$ ) for spin waves propagating in the waveguide to the left (mode $\psi_{\mathrm{L}}$ ) and to the right (mode $\psi_{\mathrm{R}}$ ). The strengths of coupling between the modes are $\zeta\left(\psi_{\mathrm{L}}\right.$ and $\left.\psi_{\mathrm{R}}\right), \Delta_{\mathrm{R}}\left(\varphi\right.$ and $\left.\psi_{\mathrm{R}}\right)$, and $\Delta_{\mathrm{L}}(\varphi$ and $\psi_{\mathrm{L}}$ ). (b) The dispersion relation of spin waves in the waveguide is shown, as computed from the results of the micromagnetic simulations. The brighter color corresponds to higher spin-wave amplitude. (c) The FFT spectrum of the stand-alone chiral resonator's average response to uniform excitation is shown on a log scale. The rectangular boxes in panels $(b, c)$ show the spectral range addressed in this paper. The two arrows in panel (c) indicate where absorption peaks were observed in scattering simulations. The inset shows the spatial distribution [in the resonator's $(x, z)$ cross section] of the FFT amplitude (by gray scale with black and white corresponding to zero and maximum, respectively) and phase (color scale shown) at the frequency of the only peak in the spectrum. Both the FFT spectrum and the mode profile were calculated from the $z$ component of the dynamic magnetization.

and its conclusions are expected to be valid for other spinwave geometries [15-20]. The same concerns the observation of the chiral scattering from the magnon dark mode of the resonator.

The paper is organized as follows. In Sec. II, we describe the methods and main results of our numerical micromagnetic simulations. In Sec. III, we describe the phenomenological model and main results of its application to the analysis of the micromagnetic simulations. Sections IV and V are then devoted to discussing the origin of the chiral coupling and to conclusions, respectively.

\section{NUMERICAL MICROMAGNETIC SIMULATIONS}

\section{A. Methods}

Micromagnetic simulations are performed for the system shown in Fig. 1(a), using MUMAX3 software [21]. The resonator is represented by a magnetic stripe with $x \times z$ cross-section dimensions of $50 \times 20 \mathrm{~nm}^{2}$. It is placed under a thin-film magnonic waveguide with $x \times z$ cross-section dimensions of $10240 \times 20 \mathrm{~nm}^{2}$. The edge to edge spacing between the resonator and waveguide is varied from 5 to $20 \mathrm{~nm}$ in steps of $5 \mathrm{~nm}$. Both the resonator and waveguide are considered infinite in the $y$ direction, which is achieved by applying periodic boundary conditions to an $80 \mathrm{~nm}$ long portion of the sample. The sample is discretized into cubic cells of $5 \mathrm{~nm}$ size. Both the waveguide and resonator have identical magnetic parameters: the saturation magnetization of 800 $\mathrm{kA} / \mathrm{m}$, the exchange constant of $13 \mathrm{pJ} / \mathrm{m}$, the Gilbert damping constant of 0.005 , and zero magnetocrystalline anisotropy; the default value of the gyromagnetic ratio is used. The stripe is magnetized in the $y$ direction due to its shape anisotropy (no bias field). The thin-film waveguide is magnetized along the $x$ direction by a bias magnetic field of $0.1 \mathrm{~T}$, confined to the thin film only. In practice, the orthogonal magnetization of the resonator and waveguide can be achieved by combining materials with different coercivities [17] or by patterning the waveguide to give it a shape anisotropy [11]. In the dynamic simulations, the sample is excited by a dynamic magnetic field $\boldsymbol{h} \| \hat{\boldsymbol{z}}$ with a spatiotemporal profile to suit the aim of each specific numerical experiment [22], while ensuring that only linear spin waves are excited. To calculate the spin-wave dispersion, the stand-alone waveguide is excited by a dynamic field that has a sinc-function profile both in time (cutoff frequency 25 $\mathrm{GHz}$ ) and along the $x$ direction (cutoff wave number $0.2 \pi$ $\mathrm{rad} / \mathrm{nm}$ ). The dynamic field is uniform in the $z$ direction. For each pair of $(y, z)$ values in the waveguide's cross section, we apply two-dimensional (2D) fast Fourier transform (FFT) in the time domain and $x$ direction to the simulated data. By summing the calculated FFT amplitudes over the waveguide's $(y, z)$ cross section for each pair of frequency $f$ and wave number $k_{x}$ values, we obtain the $f\left(k_{x}\right)$ dispersion depicted in Fig. 1(b). This dispersion has a shape characteristic for backward volume spin waves, as in in Ref. [11]. Figure 1(c) shows the spectrum of the stand-alone resonator excited by a dynamic field uniform in space and having the sinc-function profile in time. The spectrum shows the only resonance peak at $12.8 \mathrm{GHz}$, due to the quasiuniform mode [see the inset in Fig. 1(c) for its spatial profile].

To study the scattering of propagating spin waves from the resonator, we launch spin waves toward the resonator and then compute the FFT amplitude and phase for the transmitted and reflected waves [Fig. 1(a)]. The spin waves are launched as wave packets using a dynamic magnetic field of the form [23]

$$
h=h_{0} \exp \left[-\frac{\left(x \pm x_{0}\right)^{2}}{2 \sigma_{x}^{2}}\right] \exp \left[-\frac{\left(t-t_{0}\right)^{2}}{2 \sigma_{t}^{2}}\right] \cos \left( \pm k_{0} x-2 \pi f_{0} t\right)
$$

where the spatial and temporal envelopes are chosen so as to excite a narrow band of frequencies and wave numbers around the desired central values while also being able to define and trace the packets' positions in time and space. Specifically, $f_{0}$ is the central frequency of the wave packet and $k_{0}$ is the corresponding wave number, obtained from the dispersion relation shown in Fig. 1(b). The initial distance between the packet and the resonator is $x_{0}=1.75 \mu \mathrm{m}$, while its time position relative to the beginning of the simulation is $t_{0}=5.5 \mathrm{~ns}$. The plus and minus signs in front of the wave number, $\pm k_{0}$, in Eq. (1) correspond to wave packets incident on the resonator from the left- and right-hand sides, respectively, and launched at $\mp x_{0}$. The incident and scattered wave packets are sampled at $1 \mu \mathrm{m}$ from the nanomagnet. The size and duration of the 

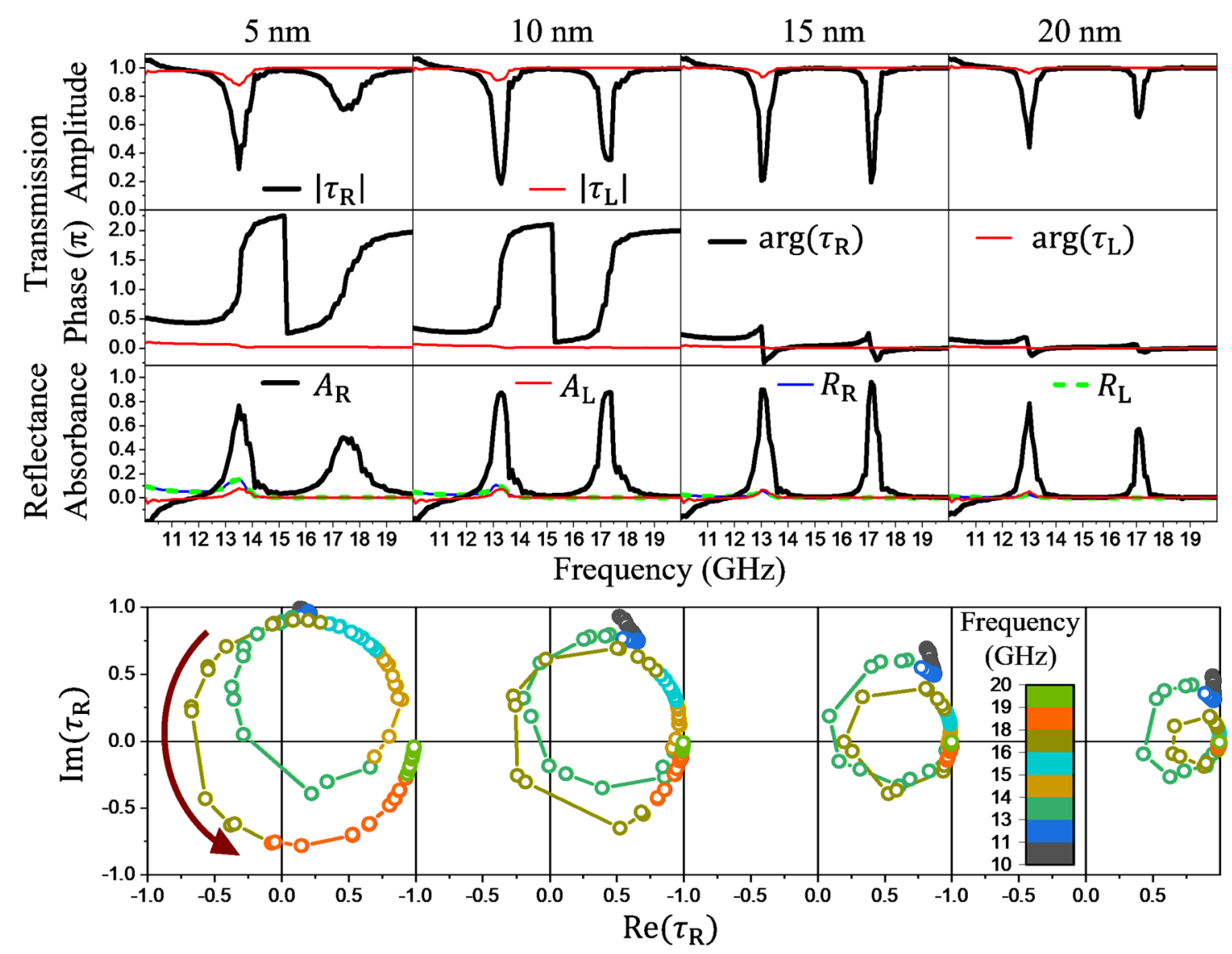

FIG. 2. The simulated scattering and absorption coefficients of the right- and left-going spin waves are shown as a function of their frequency $f_{0}$ for different values (indicated for each column) of the edge to edge separation between the waveguide and resonator. The top two rows show the magnitude and phase of the transmission coefficients $\tau_{\mathrm{R}(\mathrm{L})}$. The third row compares the absorbance $A_{\mathrm{R}(\mathrm{L})}$, and reflectance $R_{\mathrm{R}(\mathrm{L})}$. The reflectance curves for the counterpropagating waves lie on top of each other. The bottom row shows the transmission coefficient in the complex plane, plotted using $f_{0}$ as a parameter with the arrow indicating the direction in which $f_{0}$ increases.

wave packet are defined by parameters $\sigma_{x}=0.35 \mu \mathrm{m}$ and $\sigma_{t}=0.9 \mathrm{~ns}$, which yield the full width at half maximum of about $0.82 \mu \mathrm{m}$ along $x$ and just over $2 \mathrm{~ns}$ in the time domain, respectively.

The simulations and associated analyses are performed for wave packets with different central frequencies and propagation directions. Throughout the paper, subscripts $\mathrm{R}$ and $\mathrm{L}$ denote quantities corresponding to the right- and left-going spin waves, incident on the resonator from the left and from the right, respectively. The change of the incidence direction is equivalent to flipping the magnetization of the resonator [11]. To exclude the effect of propagation decay, the complex transmission, $\tau_{\mathrm{R}(\mathrm{L})}$, (reflection, $r_{\mathrm{R}(\mathrm{L})}$ ) coefficients are evaluated at each frequency as a ratio of the transmitted (reflected) complex FFT amplitudes obtained from simulations with the resonator and the transmitted complex amplitude from a reference simulation without the resonator. In each case, the compared reflected and/or transmitted waves travel the same distance and therefore experience the same propagation decay and phase delay. The reflected and incident waves are separated in the reciprocal space [23]. The absorbance and reflectance are calculated as $A_{\mathrm{R}(\mathrm{L})}=1-\left|\tau_{\mathrm{R}(\mathrm{L})}\right|^{2}-\left|r_{\mathrm{R}(\mathrm{L})}\right|^{2}$ and $R_{\mathrm{R}(\mathrm{L})}=\left|r_{\mathrm{R}(\mathrm{L})}\right|^{2}$. The results of the calculations are shown in Fig. 2. Evidently, our method of analysis works well, except for the frequencies below $11 \mathrm{GHz}$ at which the transmission coefficient's magnitude exceeds unity. The latter is attributed to a combined effect of numerical error and magnetic nonlinearity. Figure 3 shows a selection of spatial profiles, calculated using standard procedures [22], of the amplitude and phase of the dynamic magnetization in the resonators excited by the incident spin-wave packets.

\section{B. Numerical results}

We focus on the chiral modulation of the transmitted spin waves' amplitude and phase, which are most relevant to perceived spin-wave devices of magnonics. The numerically calculated frequency dependence of the magnitude of the transmission coefficient for right-going spin waves (Fig. 2, top row) shows two pronounced dips at 13.5 and 17.4 GHz. The dips are associated with modes that have quasiuniform $(13.5 \mathrm{GHz})$ and nonuniform $(17.4 \mathrm{GHz})$ profiles in the resonator (Fig. 3). The quasiuniform mode's frequency is somewhat higher than the value of $12.7 \mathrm{GHz}$ found for the stand-alone resonator [Fig. 1(c)], with the difference decreasing with increasing of the resonator to waveguide spacing. This mode is similar to that responsible for the spin-wave amplitude and phase modulation reported in Ref. [11]. The 


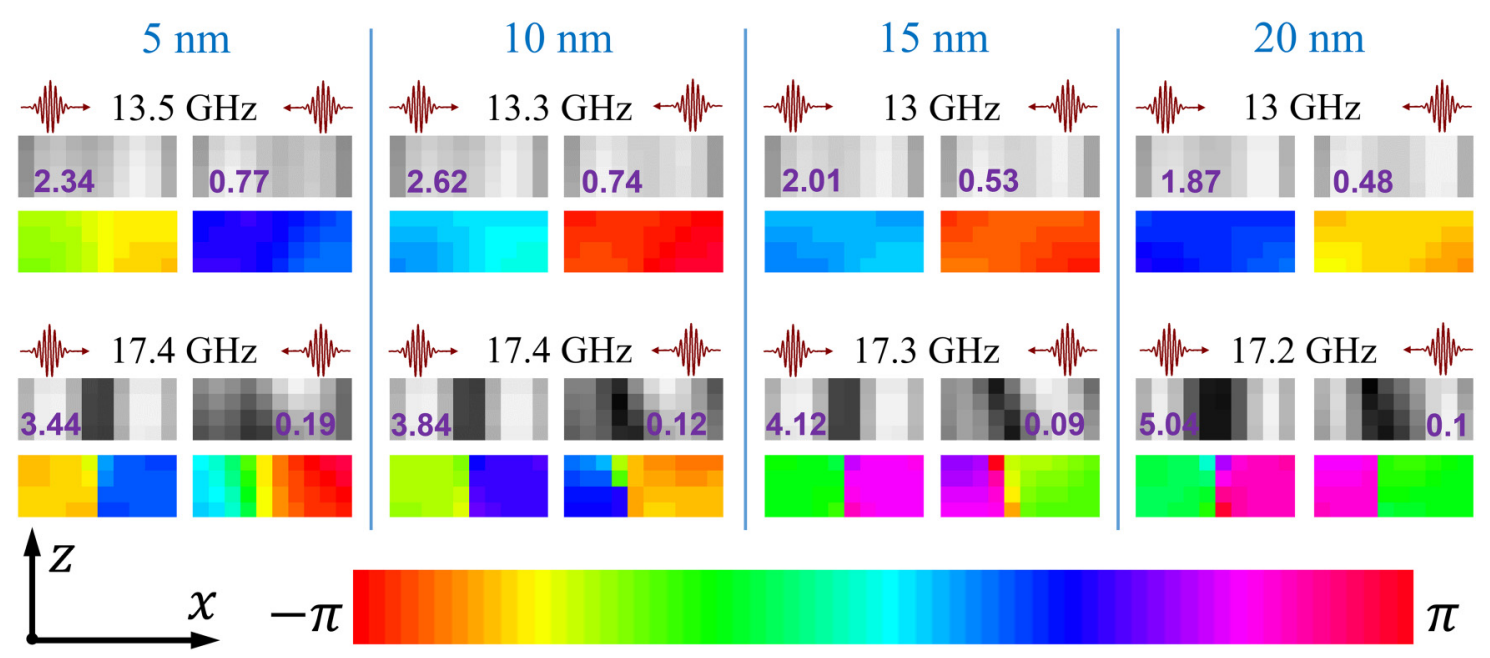

FIG. 3. The $(x, z)$ distributions of the Fourier amplitude and phase calculated from the simulated $z$ component of the dynamic magnetization in the resonator excited by right- and left-going spin waves (as indicated) are shown for the stated values of the excitation frequency and of the edge to edge separation between the waveguide and resonator. The frequencies correspond to the dips in the spin-wave transmission in Fig. 2. Black and white in the amplitude profiles correspond to zero and maximum amplitude, respectively; the different gray scales are normalized by different factors, as indicated.

nonuniform mode (not observed in Ref. [11]) represents a "dark mode," i.e., a mode that cannot be excited by a uniform dynamic magnetic field [Fig. 1(c)]. In the case of Fig. 2, its excitation is enabled by the nonuniformity of the stray magnetic field due to the incident propagating spin wave. The transmission amplitude dip at $13.5 \mathrm{GHz}$ is drastically shallower for left-going spin waves, while the dark mode appears to cause no effect on their transmission at all. Similarly, the phase of the transmission coefficient (Fig. 2, second row) is barely affected for left-going waves. For right-going waves, a phase shift of $2 \pi$ is accumulated as the spin-wave frequency crosses each of the resonator's modes at spacings of 5 and $10 \mathrm{~nm}$; a phase shift of $\pi$ is found at resonance, when the frequencies of the propagating and resonator's modes match. In contrast, for 15 and $20 \mathrm{~nm}$ spacings, the phase remains relatively close to zero.

The dependence of the magnitude and phase of the transmission coefficient on the resonator-waveguide separation is best visualized when it is plotted in the complex plane, using the spin-wave frequency as a parameter (Fig. 2, bottom row). The complex transmission coefficient makes counterclockwise loops at each resonance. For right-going waves, the loop size decreases as the resonator-waveguide separation increases. For left-going waves, the loops shrink almost to a point at $\tau_{\mathrm{L}} \cong 1$ (not shown). This observation prompts us to associate the loop size with the strength of the interaction between the waveguide and resonator. Surprisingly, the strongest interaction in our system is found for the dark mode resonance at $5 \mathrm{~nm}$ separation. The depth of modulation of the transmitted wave's amplitude increases as the loop approaches the origin. The character of modulation of the transmitted wave's phase differs qualitatively depending on whether the origin is contained by the loop. At last, we note that the enhanced apparent width of the dip and the slowed down phase variation seen at $5 \mathrm{~nm}$ separation are signatures of the loop's size becoming especially large. When this occurs, the loop center approaches the origin, and the frequency variation does not lead to any significant variation of the transmitted wave's amplitude.

In addition to the distinction between the quasiuniform and dark modes noted earlier, the profiles shown in Fig. 3 reveal that the precession amplitude in the resonator is consistently higher for its excitation by right-going waves as compared to the left-going ones: by a factor of 3-4 for the quasiuniform mode and 20-50 for the dark mode. This is consistent with the chirality of the frequency dependence of the spin-wave scattering coefficients shown in Fig. 2. The precession amplitude does not, however, vary monotonically as the spacing between the resonator and waveguide increases. This points to a resonant origin of the scattering, as discussed in the following section.

\section{PHENOMENOLOGICAL MODEL}

\section{A. Assumptions and the key relations}

The salient features of the chiral resonant scattering of spin waves in our system [Fig. 1(a)] are captured by the following model. The resonator (located at $x=0$ ) affects the propagating plane-wave modes in two ways. Firstly, there could be nonresonant scattering between the waves propagating to the right, $\psi_{\mathrm{R}}(t, x)$, and to the left, $\psi_{\mathrm{L}}(t, x)$, with group velocity $v$. We characterize this scattering by the coupling constants $\zeta$ and $\zeta^{*}$, where the asterisk denotes complex conjugation. Secondly, the resonator's mode, $\varphi(t)$, can hybridize with the propagating modes, which we describe using its complex coupling strengths to the right, $\Delta_{R}$, and left, $\Delta_{\mathrm{L}}$, propagating modes. These coupling strengths depend on, among other factors, the resonator size, the resonant mode character, and the propagating mode wavelength. Their calculation is, however, beyond the scope of this paper. Both resonant and off-resonant scattering processes can be described as reemission of scattered waves by pointlike source terms proportional to the delta function $\delta(x)$. Mathematically, this is encompassed by the 

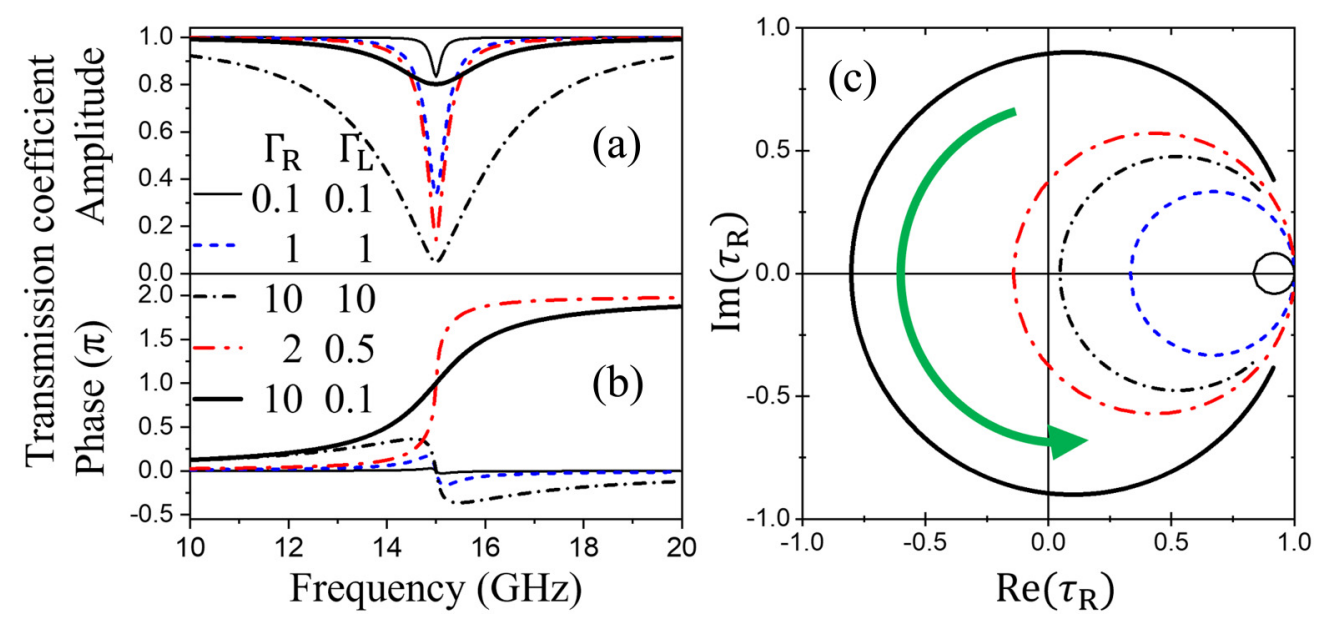

FIG. 4. The magnitude and phase of the transmission coefficient given by Eq. (5) for the right-going waves are shown as a function of their frequency, $f=\omega / 2 \pi$, in panels (a,b), respectively. The values of the radiative linewidths $\Gamma_{\mathrm{R}}$ and $\Gamma_{\mathrm{L}}$ are indicated as fractions of the resonator's dissipative linewidth $\Gamma_{0}=2 \pi \times 0.5 \mathrm{GHz}$, while its resonance frequency is $\Omega_{0}=2 \pi \times 15 \mathrm{GHz}$. (c) The dependences from (a,b) are plotted in the complex plane, using $f$ as a parameter with the arrow indicating the direction in which $f$ increases.

following system of scalar differential equations:

$$
\begin{gathered}
\frac{\partial \psi_{\mathrm{R}}(t, x)}{\partial t}+v \frac{\partial \psi_{\mathrm{R}}(t, x)}{\partial x}=-i \delta(x)\left[\zeta \psi_{\mathrm{L}}(t, 0)+\Delta_{\mathrm{R}} \varphi(t)\right], \\
\frac{\partial \psi_{\mathrm{L}}(t, x)}{\partial t}-v \frac{\partial \psi_{\mathrm{L}}(t, x)}{\partial x}=-i \delta(x)\left[\zeta^{*} \psi_{\mathrm{R}}(t, 0)+\Delta_{\mathrm{L}} \varphi(t)\right], \\
\frac{\partial \varphi(t)}{\partial t}+i\left(\Omega_{0}-i \Gamma_{0}\right) \varphi(t)=-i\left[\Delta_{\mathrm{L}}^{*} \psi_{\mathrm{L}}(t, 0)+\Delta_{\mathrm{R}}^{*} \psi_{\mathrm{R}}(t, 0)\right],
\end{gathered}
$$

where $\Omega_{0}$ and $\Gamma_{0}$ are the angular frequency and dissipative linewidth of the scatterer; and in the small frequency region around the resonance, we treat the dispersion as linear and define the angular frequency $\omega$ and wave number $k$ of the plane waves relative to their resonance values, i.e.,

$$
\omega=\Omega_{0}+v\left(k-k_{0}\right)
$$

where $k_{0}$ is the wave number corresponding to $\Omega_{0}$.

Integrating Eq. (2), we obtain the following system of algebraic equations connecting the plane waves on the opposite sides from the scatterer:

$$
\begin{aligned}
+i v\left[\psi_{\mathrm{R}}\right] & =\zeta\left\langle\psi_{\mathrm{L}}\right\rangle+\Delta_{\mathrm{R}} \varphi, \\
-i v\left[\psi_{\mathrm{L}}\right] & =\zeta^{*}\left\langle\psi_{\mathrm{R}}\right\rangle+\Delta_{\mathrm{L}} \varphi, \\
\left(\omega-\Omega_{0}+i \Gamma_{0}\right) \varphi & =\Delta_{\mathrm{L}}^{*}\left\langle\psi_{\mathrm{L}}\right\rangle+\Delta_{\mathrm{R}}^{*}\left\langle\psi_{\mathrm{R}}\right\rangle,
\end{aligned}
$$

where $\left[\psi_{\mathrm{R}(\mathrm{L})}\right] \equiv \psi_{\mathrm{R}(\mathrm{L})}(+0)-\psi_{\mathrm{R}(\mathrm{L})}(-0)$ denotes the discontinuity of the plane-wave solutions at $x=0$, at which we have regularized the solution as $\left\langle\psi_{\mathrm{R}(\mathrm{L})}\right\rangle \equiv$ $\frac{1}{2}\left[\psi_{\mathrm{R}(\mathrm{L})}(+0)+\psi_{\mathrm{R}(\mathrm{L})}(-0)\right]$. In our case, $\left[\psi_{\mathrm{R}}\right]=\tau_{\mathrm{R}}-1$, $\left[\psi_{\mathrm{L}}\right]=-r_{\mathrm{R}},\left\langle\psi_{\mathrm{R}}\right\rangle=\frac{1}{2}\left(1+\tau_{\mathrm{R}}\right)$, and $\left\langle\psi_{\mathrm{L}}\right\rangle=\frac{1}{2} r_{\mathrm{R}}$ for rightpropagating waves (incident from the left) and $\left[\psi_{\mathrm{R}}\right]=r_{\mathrm{L}}$, $\left[\psi_{\mathrm{L}}\right]=1-\tau_{\mathrm{L}},\left\langle\psi_{\mathrm{R}}\right\rangle=\frac{1}{2} r_{\mathrm{L}}$, and $\left\langle\psi_{\mathrm{L}}\right\rangle=\frac{1}{2}\left(1+\tau_{\mathrm{L}}\right)$ for leftpropagating waves (incident from the right). The resulting equations are readily solved to yield the reflection, $r_{\mathrm{R}(\mathrm{L})}$, and transmission, $\tau_{\mathrm{R}(\mathrm{L})}$, coefficients, as well as absorbance $R_{\mathrm{R}(\mathrm{L})}$ and reflectance $A_{\mathrm{R}(\mathrm{L})}$. For the case of dominant resonant scat- tering $\zeta=0$ we obtain

$$
\begin{aligned}
\tau_{\mathrm{R}} & =\frac{\omega-\Omega_{0}+i\left(\Gamma_{0}-\Gamma_{\mathrm{R}}+\Gamma_{\mathrm{L}}\right)}{\omega-\Omega_{0}+i \Gamma_{\mathrm{tot}}}, \\
r_{\mathrm{R}} & =-\frac{i}{v} \frac{\Delta_{\mathrm{R}}^{*} \Delta_{\mathrm{L}}}{\omega-\Omega_{0}+i \Gamma_{\mathrm{tot}}}, \\
A_{\mathrm{R}} & =\frac{4 \Gamma_{\mathrm{R}} \Gamma_{0}}{\left(\omega-\Omega_{0}\right)^{2}+\Gamma_{\mathrm{tot}}^{2}},
\end{aligned}
$$

where $\Gamma_{\mathrm{R}(\mathrm{L})} \equiv\left|\Delta_{\mathrm{R}(\mathrm{L})}\right|^{2} /(2 v)$ are the radiative linewidths into the right- (left-) propagating plane waves, and $\Gamma_{\text {tot }}=\Gamma_{0}+$ $\Gamma_{\mathrm{R}}+\Gamma_{\mathrm{L}}$ is the total linewidth of the resonator. The corresponding expressions for the left-going waves are obtained from Eq. (5) by swapping the subscripts $\mathrm{R}$ and $\mathrm{L}$.

\section{B. Application to analysis of the numerical results}

Figure 4 presents the frequency dependence of the complex transmission coefficient calculated using Eq. (5). We find that the phenomenological model supports fully the qualitative conclusions made earlier based on the results of the numerical simulations (Fig. 2). In addition, the theory reveals the key role played by the chirality of the coupling between the propagating spin waves and the resonator's modes. Both Eq. (5) and Fig. 3 show that, although reciprocal (nonchiral, i.e., $\Gamma_{R}=\Gamma_{L}$ ) resonators can lead to a significant modulation of the transmission, they fall short of the performance of chiral resonators. For instance, zero transmission can only be achieved when the condition $\Gamma_{\mathrm{R}}=\Gamma_{0}+\Gamma_{\mathrm{L}}$ is fulfilled. The device in this regime was tagged a "spin-wave valve" in Ref. [11], but it may be also appropriate to call it a magnonic diode [24-26]. Our simulations suggest that this behavior can be achieved using chiral resonators with relatively high damping, e.g., as that in metallic ferromagnets. At the damping constant of 0.005 , the coupling strength required for our system to behave as a good magnonic diode is achieved already at the waveguide-resonator spacings of 10 and $15 \mathrm{~nm}$. The dissipative linewidth of the resonator can be controlled further using spin currents [27]. 


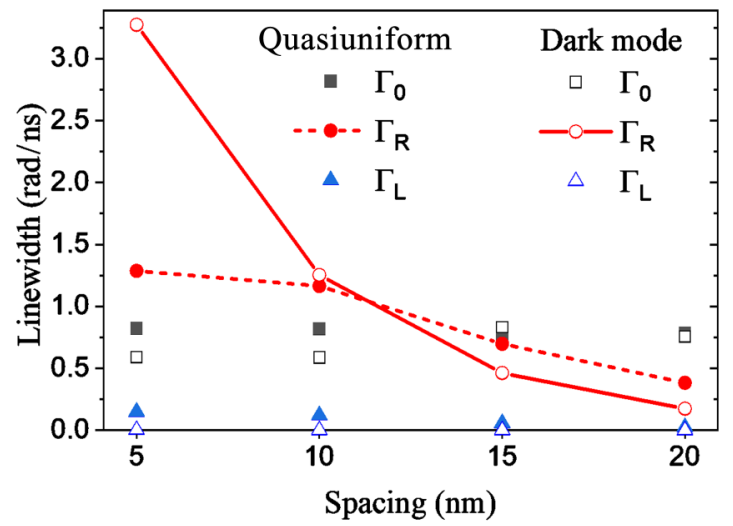

FIG. 5. The dissipative, $\Gamma_{0}$, and radiative, $\Gamma_{\mathrm{R}(\mathrm{L})}$, linewidths extracted from the fitting of the numerical data to Eq. (5) are presented for the quasiuniform and dark modes as a function of the resonator to waveguide spacing and the direction of spin-wave incidence.

According to Eq. (5), the use of the resonator as a $\pi$ phase shifter imposes much stricter requirements on its chirality, damping, and coupling strength (Fig. 4). This is because the reduction of the transmitted amplitude is a parasitic effect in this case. Nonetheless, the dark mode at $5 \mathrm{~nm}$ separation enables at least half of the spin-wave power to go through while shifting the phase by $\pi$. This is comparable to the result achieved in the "spin-wave phase shifter" from Ref. [11], using a stripe waveguide and a "pointed" resonator. Potentially, even better performance could be achieved using an antiparallelly magnetized waveguide and resonator in the Damon-Eshbach geometry [18]. However, the magnetization of the waveguide in the direction of the spin-wave propagation exploited here and in Ref. [11] better suits the goal of creation of bias-free magnonic circuitry [11]. Such phase shifters placed in the two arms of a Mach-Zehnder interferometer yield an XNOR gate, as presented in Ref. [28] and reproduced in Ref. [27]. By placing two resonators on the same straight magnonic waveguide, a magnonic NAND gate is formed [28].

Equation (5) predicts that the reflectance from a chiral resonator (with, e.g., $\Gamma_{\mathrm{R}} \gg \Gamma_{\mathrm{L}}$ ) must be both weak and reciprocal. The numerical simulations confirm this prediction. Indeed, the third row of Fig. 2 shows that most of the incident spin-wave power not transmitted by the resonator is absorbed rather than reflected. This resonant absorption of the spin-wave energy was observed in the "spin-wave valve" in Ref. [11] and labeled "magnon trapping" in Ref. [18].

The scattering line shapes shown in both Figs. 2 and 4 are rather symmetric relative to the resonant frequency. The theory shows that this is consistent with the weakness of the nonresonant contributions to the scattering between the rightand left-going waves. Allowing the nonresonant coupling constants $\zeta$ and $\zeta^{*}$ to have finite values, one would recover the Fano resonance and its characteristic asymmetric line shapes.

Figure 5 and Table I present results of fitting the numerical transmission coefficient and absorbance from Fig. 2 to Eq. (5), augmented with additional background (see Supplemental Material [29]) to offset (at least, partly) spurious backgrounds whose origins are not captured by the model. The fitted values of the radiative linewidths exhibit a strong chirality for all resonator to waveguide spacings, which is also reflected in the value of the nonreciprocity parameter $\eta$, defined as [17]

$$
\eta=\frac{\Gamma_{\mathrm{R}}-\Gamma_{\mathrm{L}}}{\Gamma_{\mathrm{R}}+\Gamma_{\mathrm{L}}} \times 100 \% .
$$

The nonreciprocity parameter consistently approaches $100 \%$ for the dark mode, albeit remaining at about $80 \%$ for the quasiuniform mode. The radiative linewidths and therefore the coupling strength consistently decrease as the spacing increases. This dependence is stronger for the dark mode, as expected due to its nonuniformity. Remarkably, the dark mode at $5 \mathrm{~nm}$ spacing outperforms the quasiuniform mode in terms of both the coupling strength and chirality, which is consistent with our observations made from Fig. 2 earlier. The variation of the resonance frequency and dissipative linewidth as a function of the spacing are due to reasons not captured by our model and will be investigated elsewhere.

\section{ORIGIN OF THE CHIRAL COUPLING}

Our simulations and their analysis via the model provide a strong evidence for the chirality of the coupling between the resonator and spin-wave modes in the film. However, the model merely postulates the chiral coupling and shows how this chirality explains our numerical results. Hence, we now discuss the origin of the chiral coupling between the resonator's modes and propagating spin waves. This can be

TABLE I. The computer fitted values of the resonance frequencies; dissipative, $\Gamma_{0}$, and radiative, $\Gamma_{\mathrm{R}(\mathrm{L})}$, linewidths; and nonreciprocity parameter $\eta$ are shown for the quasiuniform and dark modes and different values of the resonator to waveguide spacing. Each column corresponds to a separate independent fit. In some cases, the value of $\Gamma_{\mathrm{L}}$ was too small for automated fitting and therefore had to be adjusted manually and fixed prior to the computerized finding values of the other parameters.

\begin{tabular}{|c|c|c|c|c|c|c|c|c|}
\hline \multirow[b]{2}{*}{ Spacing } & \multicolumn{4}{|c|}{ Quasiuniform mode } & \multicolumn{4}{|c|}{ Dark mode } \\
\hline & $5 \mathrm{~nm}$ & $10 \mathrm{~nm}$ & $15 \mathrm{~nm}$ & $20 \mathrm{~nm}$ & $5 \mathrm{~nm}$ & $10 \mathrm{~nm}$ & $15 \mathrm{~nm}$ & $20 \mathrm{~nm}$ \\
\hline$\frac{\Omega_{0}}{2 \pi}(\mathrm{GHz})$ & 13.56 & 13.26 & 13.07 & 12.97 & 17.49 & 17.25 & 17.11 & 17.08 \\
\hline$\frac{\Gamma_{0}}{2 \pi}(\mathrm{GHz})$ & 0.13 & 0.13 & 0.12 & 0.12 & 0.09 & 0.09 & 0.13 & 0.12 \\
\hline$\frac{\Gamma_{\mathrm{R}}}{2 \pi}(\mathrm{GHz})$ & 0.21 & 0.18 & 0.11 & 0.06 & 0.52 & 0.20 & 0.07 & 0.028 \\
\hline$\frac{\Gamma_{\mathrm{L}}}{2 \pi}(\mathrm{GHz})$ & $\begin{array}{c}0.024 \\
\text { Fixed }\end{array}$ & 0.019 & 0.010 & $\begin{array}{c}0.004 \\
\text { Fixed }\end{array}$ & $\begin{array}{l}0.0003 \\
\text { Fixed }\end{array}$ & $\begin{array}{l}0.00008 \\
\text { Fixed }\end{array}$ & $\begin{array}{c}0.00004 \\
\text { Fixed }\end{array}$ & $\begin{array}{l}0.000008 \\
\text { Fixed }\end{array}$ \\
\hline$\eta$ & $79.1 \%$ & $81.2 \%$ & $84.8 \%$ & $87.7 \%$ & $99.9 \%$ & $99.9 \%$ & $99.9 \%$ & $99.9 \%$ \\
\hline
\end{tabular}



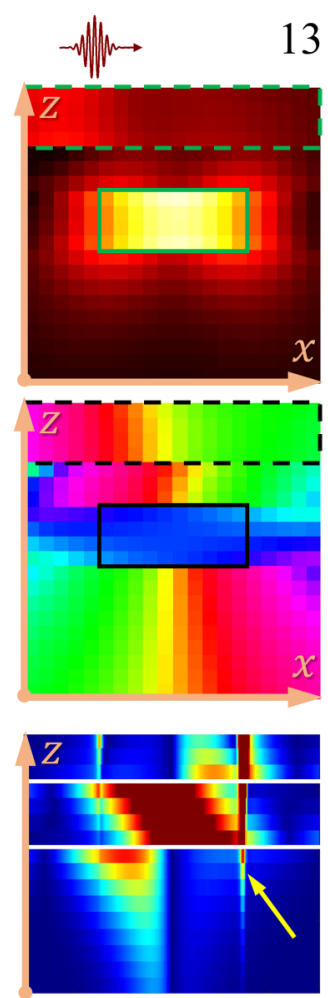

$-100-50 \quad 0 \quad 50 \quad 100$

Wave number $(\mathrm{rad} / \mu \mathrm{m})$

\section{$\mathrm{GHz}$}
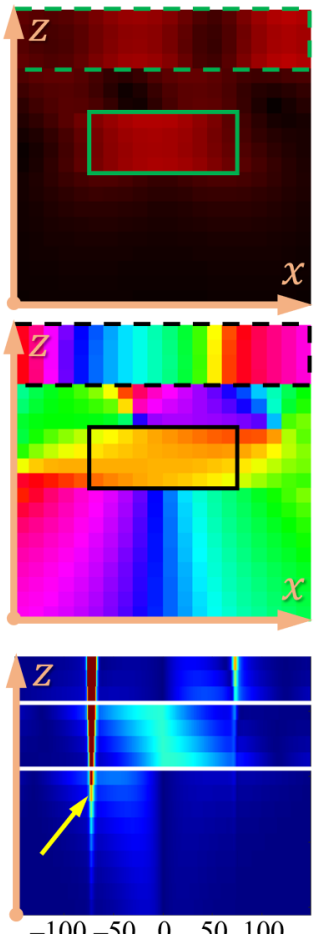

$-100-50 \quad 0 \quad 50 \quad 100$

Wave number $(\mathrm{rad} / \mu \mathrm{m})$
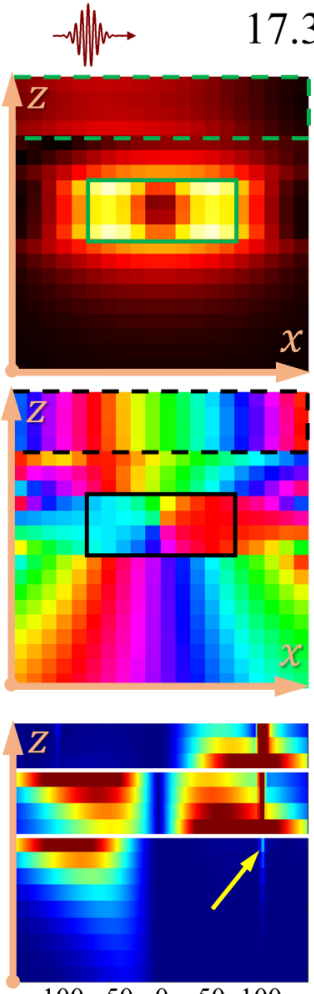

$-100-50 \quad 0 \quad 50 \quad 100$

Wave number $(\mathrm{rad} / \mu \mathrm{m})$
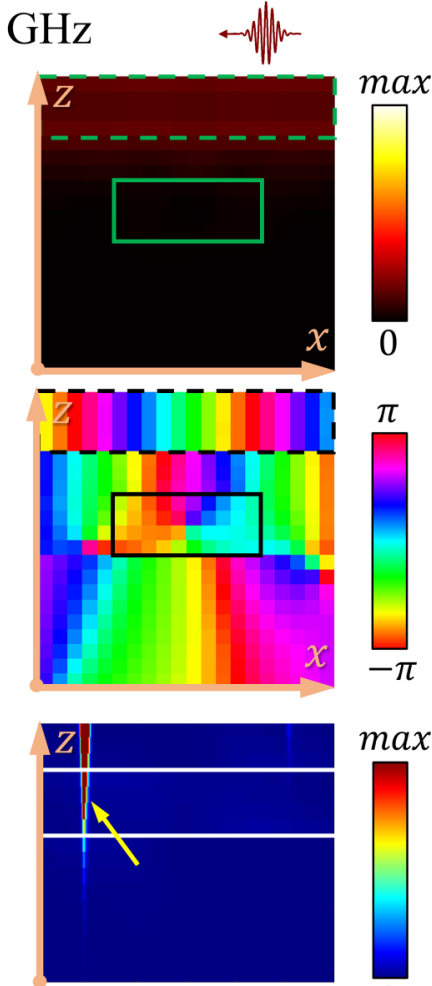

$-100-50 \quad 0 \quad 50 \quad 100$

Wave number $(\mathrm{rad} / \mu \mathrm{m})$

FIG. 6. The real and reciprocal space distributions of the stray magnetic field at frequencies of 13 and $17.3 \mathrm{GHz}$ are shown for the resonator to waveguide spacing of $15 \mathrm{~nm}$. At each frequency, the left and right columns correspond to excitation by right- and left-going spin-wave packets, respectively. The top and middle rows show real-space distributions of the field's Fourier amplitude and phase, respectively. The bottom row shows the corresponding reciprocal space distribution of the Fourier amplitude, calculated for the whole structure rather than just its part shown above. The dashed and solid outlines show the waveguide's ( $20 \mathrm{~nm}$ thickness) and resonator's $(20 \mathrm{~nm} \times 50 \mathrm{~nm}) \mathrm{cross}$ sections, respectively. The yellow arrows in the bottom row point to the vertical features due to the field from the incident spin waves.

done in either of two ways: (i) one may consider the Zeeman energy of spin waves' dynamical magnetization in the resonator's dynamic stray field. Equivalently, the coupling is given by (ii) the energy of the resonator's magnetization in the spin waves' stray field, which coincides with (i) due to the magnetic reciprocity theorem [30]. Here, we use approach (i), also implemented, e.g., in Refs. [11,12], while we refer the reader to Refs. [17,18,31] for a systematic discussion of approach (ii). To elucidate the origin of the chirality in the stray field induced in the waveguide by the resonator, it is helpful to Fourier transform the field. The coupling of the field to a propagating spin-wave mode is proportional to the field's Fourier amplitude for a wave number coinciding, both in magnitude and sign, with that of the propagating mode. A chiral coupling then manifests itself as an asymmetric distribution of the field's Fourier amplitude for opposite signs of the wave number [11]. This distribution is reversed relative to the $k_{x}=0$ axis when the magnetization of the resonator and therefore the chirality of its precession are reversed.

Figure 6 shows, in the real and reciprocal space, the Fourier amplitudes of the $h_{z}$ component of the stray magnetic field from the resonator driven by spin waves with frequencies corresponding to the absorption peaks due to the quasiuniform (left two columns) and dark (right two columns) modes.
In addition, the distributions include contributions from the field due to the incident spin waves themselves. To evaluate the chirality, we need to compare the field distributions for excitation by right- and left-going spin waves, shown in the left and right columns, respectively, at each frequency. In the real space, the amplitude plots (top row) show the expected asymmetry between the two incidence directions, with the right-going waves exciting the resonators drastically more efficiently. This asymmetry can be explained by considering the field's phase variation (middle row). In the space below the resonator, the direction of this phase variation is dominated by the chirality of the resonator's precession, which is always the same. In the space between the waveguide and resonator, the fields from the spin waves and resonator mix, making up a more complex distribution. Yet, for excitation by right-going waves, this distribution is dominated by the resonator's field, and we see that the phase variation is opposite to that below the resonator. Hence, the strong coupling occurs when, in the waveguide, this phase variation matches that in the incident spin waves, which is the case for excitation by right-going waves. In contrast, the coupling of the resonator's field to the left-going waves is weak since the directions of the phase variation of the field and the spin waves do not match. The argument is even more evident when considered in the reciprocal space (bottom row). The Fourier amplitude 

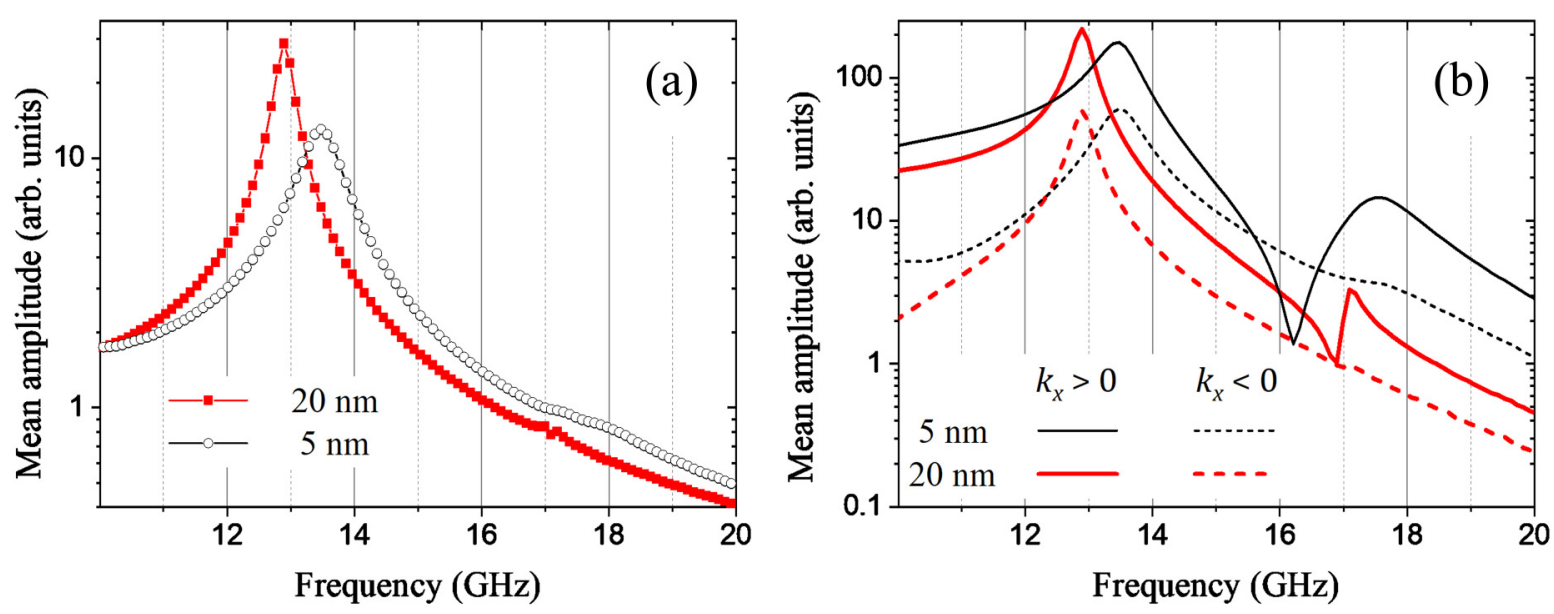

FIG. 7. (a) The FFT spectrum of the resonator's response to uniform excitation is shown for the resonator to waveguide spacings of 5 and $20 \mathrm{~nm}$. (b) The spectra of spin waves emitted in the two opposite directions as a result of the excitation in (a) are shown.

of the resonator's field is concentrated in the negative (positive) half of the reciprocal space below (above) the resonator. Hence, the stronger coupling occurs when the wave number of the spin waves is positive, i.e., for the right-going spin waves.

The pattern seen in Fig. 6 can be explained via the following argument. Let $\phi_{k}(x, z)$ be the Fourier harmonic of the magnetostatic potential induced by an individual magnetic charge within the resonator, and $k$ its wave number along the waveguide, i.e., $k=k_{x}$. Away from the point charge, the harmonic obeys Laplace's equation, and so its profile is given by $\phi_{k}(x, z)=\phi_{0} \exp (i k x \mp|k| z)$, with negative sign for $z>0$ and positive for $z<0$, assuming that $z=0$ corresponds to the position of the point charge. This has important consequences. Firstly, the respective components of the magnetic field $\boldsymbol{h}=-\nabla \phi_{k}(x, z)$ have the same profiles, i.e., $h_{x}=$ $-i k \phi_{k}(x, z)$ and $h_{z}= \pm|k| \phi_{k}(x, z)$. Secondly, these two field components are of the same magnitude, while their phases are shifted by $\pm 90^{\circ}$. Thus, the field harmonic has a perfect circular polarization, with the handedness defined by whether the resonator is above or below the waveguide and whether the wave number $k$ is positive or negative. This field would couple to the spin wave's magnetization components $s_{x}$ and $s_{z}$ via the term $\left(-s_{x}^{*} h_{x}-s_{z}^{*} h_{z}\right) \propto\left[i \operatorname{sign}(k) s_{x}^{*} \mp s_{z}^{*}\right]$, where the minus sign corresponds to our case of the resonator located below the waveguide. Had the receiving spin wave been itself chiral in the $x z$ plane, this would provide the chiral coupling $[17,18,31]$. However, this mechanism is not active in our geometry, in which the waveguide is magnetized collinearly to the spin-wave propagation directions.

To explain chirality in our geometry, we recall that the resonator is described by a magnetic dipole with components $m_{x}$ and $m_{z}$. Considering the dipole as a pair of opposite magnetic charges, one computes the difference of their stray fields, which brings the interaction to the symmetric form $\left[i \operatorname{sign}(k) s_{x}^{*} \mp s_{z}^{*}\right]\left[i \operatorname{sign}(k) m_{x} \mp m_{z}\right]$. Specializing to precession $m_{x}=i \varepsilon m_{z}$ of ellipticity $\varepsilon$, with handedness (and therefore the sign of $\varepsilon$ ) defined by the equilibrium magnetization in the resonator, one finds that the amplitude of the stray field component that mediates the coupling is proportional to $[\varepsilon \operatorname{sign}(k) \pm 1]$. If the precession is circular, i.e., $\varepsilon= \pm 1$, the coupling is completely suppressed for one sign of $k$, regardless of whether the spin wave itself is chiral. In our case of the resonator magnetized along the $y$ axis $(\varepsilon>0)$ and located below the waveguide $(z>0)$ that is magnetized along the $x$ axis $\left(s_{x}^{*} \equiv 0\right)$, the coupling chirality is mediated by the field component $h_{z} \propto[\varepsilon \operatorname{sign}(k)+1]$ only. This field may be drastically suppressed for negative (but not for positive) $k$ values, consistent with Fig. 6.

We see that dipolar stray fields from the resonator provide a simple and general mechanism for chiral coupling in resonant magnonic systems such as ours. In the Damon-Eshbach geometry, additional chirality arises from spin waves through the factor $\left[i \operatorname{sign}(k) s_{x}^{*} \mp s_{z}^{*}\right]$, as considered in Refs. [17,18,20,31]. A useful mathematical discussion of the coupling (including the form factor) for the case of uniform precession in resonators with rectangular cross sections can be found in Ref. [32], while resonators with circular cross sections were considered in Ref. [12]. For scattering mediated by nonuniform modes, the coupling energy must be weighted with the specific mode profile, which will determine the degree of mode chirality. The detailed analysis of this is, however, beyond the scope of this paper.

We point out that the chiral scattering discussed here is different from the nonreciprocity of the magnonic dispersion relation [33,34]. Moreover, the dispersion nonreciprocity does not guarantee nonreciprocity of the scattering coefficients [35]. At the same time, our theory predicts that a nonreciprocal dispersion relation can make the radiative linewidths unequal, $\Gamma_{\mathrm{R}} \neq \Gamma_{\mathrm{L}}$, if the group velocities of the forward and backward propagating spin waves are unequal. Then, the scattering will be chiral even when $\Delta_{R}=\Delta_{L}$.

Finally, we demonstrate that the dark modes remain such even when the resonator is placed close to the waveguide. Figure 7 shows representative spectra of the resonator itself as well as of spin waves emitted in the two opposite directions into the waveguide when the resonator is excited by a broadband pulse of uniform magnetic field. The spectra confirm the findings from Ref. [12] that the emission is chiral and that the resonator acts as a "resonant microwave to spin wave 
transducer." At the same time, the spectra show that the emission for the dark modes is at least an order of magnitude weaker than that for the quasiuniform modes; i.e., the dark modes couple only weakly to the incident microwave magnetic field.

\section{CONCLUSIONS}

In summary, we have modeled both numerically and analytically the scattering of dipole exchange spin waves from mesoscale chiral magnetic resonators. We have presented conditions for the most efficient modulation of the spin-wave amplitude and phase in such devices. The geometry in which the magnonic waveguide is magnetized collinear to the direction of spin-wave propagation is shown to be fully adequate for creation of magnonic diodes. Furthermore, the magnonic dark mode of the resonator is shown to be as good as or even to outperform the quasiuniform mode in terms of chiral trapping of incident spin waves or controlling their phase. This not only enables creation of efficient phase shifters at elevated frequencies but it also opens prospects for creation of magnonic integrated circuits based on these phenomena. Indeed, the resonators demonstrated in Refs. [11,12] and further investigated here could be used to build the three main elements of magnonic devices [2]: chiral spin-wave sources (see, e.g., the emission for the quasiuniform mode at $20 \mathrm{~nm}$ spacing, Fig. 7), chiral spin-wave detectors or diodes (see, e.g., the absorbance by the dark mode at $15 \mathrm{~nm}$ spacing, Fig. 2), and phase shifters (see, e.g., the transmission for the dark mode at $5 \mathrm{~nm}$ spacing, Fig. 2). To achieve this, one would need to ensure that the frequencies of the modes match, which could be achieved by varying the widths of the stripe resonators. Then, the decoupling of the dark modes from the incident uniform excitation shown in Fig. 7 suggests that circuits containing several such magnonic devices could be driven by the same global uniform magnetic field [12] without compromising their output.

\section{ACKNOWLEDGMENTS}

The authors gratefully acknowledge useful discussions with F. B. Mushenok, I. R. Hooper, and V. D. Poimanov at early stages of this work. The research leading to these results has received funding from the Engineering and Physical Sciences Research Council of the United Kingdom under Projects No. EP/L019876/1 and No. EP/T016574/1. The data that support the findings of this study are available from the corresponding author upon reasonable request.
[1] A. I. Akhiezer, V. G. Bar'yakhtar, and S. V. Peletminskii, Spin Waves (North-Holland, Amsterdam, 1968).

[2] V. V. Kruglyak, S. O. Demokritov, and D. Grundler, Magnonics, J. Phys. D: Appl. Phys. 43, 264001 (2010).

[3] G. Csaba, A. Papp, and W. Porod, Perspectives of using spin waves for computing and signal processing, Phys. Lett. A 381, 1471 (2017).

[4] H. Al-Wahsh, E. H. El Boudouti, B. Djafari-Rouhani, A. Akjouj, T. Mrabti, and L. Dobrzynski, Evidence of Fano-like resonances in mono-mode magnetic circuits, Phys. Rev. B 78, 075401 (2008).

[5] S. Louis, I. Lisenkov, S. Nikitov, V. Tyberkevych, and A. Slavin, Bias-free spin-wave phase shifter for magnonic logic, AIP Adv. 6, 065103 (2016).

[6] A. Janutka, Resonance of spin waves and domain-wall excitations in ferromagnetic stripes, IEEE Magn. Lett. 4, 4000104 (2013).

[7] A. V. Mikhailov and A. I. Yaremchuk, Forced motion of a domain wall in the field of a spin wave, Pis'ma Zh. Eksp. Teor. Fiz. 39, 296 (1984) [JETP Lett. 39, 354 (1984)].

[8] R. Huber, T. Schwarze, and D. Grundler, Nanostripe of subwavelength width as a switchable semitransparent mirror for spin waves in a magnonic crystal, Phys. Rev. B 88, 100405(R) (2013).

[9] A. Haldar, D. Kumar, and A. O. Adeyeye, A reconfigurable waveguide for energy-efficient transmission and local manipulation of information in a nanomagnetic device, Nat. Nanotechnol. 11, 437 (2016).

[10] Q. Wang, T. Brächer, M. Mohseni, B. Hillebrands, V. I. Vasyuchka, A. V. Chumak, and P. Pirro, Nanoscale spin-wave wake-up receiver, Appl. Phys. Lett. 115, 092401 (2019).

[11] Y. Au, M. Dvornik, O. Dmytriiev, and V. V. Kruglyak, Nanoscale spin wave valve and phase shifter, Appl. Phys. Lett. 100, 172408 (2012).
[12] Y. Au, E. Ahmad, O. Dmytriiev, M. Dvornik, T. Davison, and V. V. Kruglyak, Resonant microwave-to-spin-wave transducer, Appl. Phys. Lett. 100, 182404 (2012).

[13] N. I. Polushkin, Combined Electron Resonance Driven by an All-Oscillating Potential of Patterned Magnets, Phys. Rev. Lett. 103, 077201 (2009).

[14] The ability of discrete modes of a magnetic nanoelement to launch omnidirectional spin waves propagating in a continuous film was shown in, V. E. Demidov, S. O. Demokritov, B. Hillebrands, M. Laufenberg, and P. P. Freitas, Radiation of spin waves by a single micrometer-sized magnetic element, Appl. Phys. Lett. 85, 2866 (2004).

[15] M. Mruczkiewicz, P. Graczyk, P. Lupo, A. Adeyeye, G. Gubbiotti, and M. Krawczyk, Spin-wave nonreciprocity and magnonic band structure in a thin permalloy film induced by dynamical coupling with an array of Ni stripes, Phys. Rev. B 96, 104411 (2017).

[16] P. Graczyk, M. Krawczyk, S. Dhuey, W. G. Yang, H. Schmidt, and G. Gubbiotti, Magnonic band gap and mode hybridization in continuous permalloy films induced by vertical dynamic coupling with an array of permalloy ellipses, Phys. Rev. B 98, 174420 (2018).

[17] J. L. Chen, T. Yu, C. P. Liu, T. Liu, M. Madami, K. Shen, J. Y. Zhang, S. Tu, M. S. Alam, K. Xia, M. Z. Wu, G. Gubbiotti, Y. M. Blanter, G. E. W. Bauer, and H. M. Yu, Excitation of unidirectional exchange spin waves by a nanoscale magnetic grating, Phys. Rev. B 100, 104427 (2019).

[18] T. Yu, H. Wang, M. A. Sentef, H. Yu, and G. E. W. Bauer, Magnon trap by chiral spin pumping, Phys. Rev. B 102, 054429 (2020).

[19] K. Sobucki, W. Śmigaj, J. Rychły, M. Krawczyk, and P. Gruszecki, Resonant subwavelength control of the phase of spin waves reflected from a Gires-Tournois interferometer, Sci. Rep. 11, 4428 (2021). 
[20] H. Qin, R. B. Holländer, L. Flajšman, F. Hermann, R. Dreyer, G. Woltersdorf, and S. van Dijken, Nanoscale magnonic FabryPérot resonator for low-loss spin-wave manipulation, Nat. Commun. 12, 2293 (2021).

[21] A. Vansteenkiste, J. Leliaert, M. Dvornik, M. Helsen, F. GarciaSanchez, and B. Van Waeyenberge, The design and verification of MUMAX3, AIP Adv. 4, 107133 (2014).

[22] M. Dvornik, Y. Au, and V. V. Kruglyak, Micromagnetic simulations in magnonics, Top. Appl. Phys. 125, 101 (2013).

[23] N. J. Whitehead, S. A. R. Horsley, T. G. Philbin, and V. V. Kruglyak, A Luneburg lens for spin waves, Appl. Phys. Lett. 113, 212404 (2018).

[24] J. Lan, W. C. Yu, R. Q. Wu, and J. Xiao, Spin-Wave Diode, Phys. Rev. X 5, 041049 (2015).

[25] M. Grassi, M. Geilen, D. Louis, M. Mohseni, T. Brächer, M. Hehn, D. Stoeffler, M. Bailleul, P. Pirro, and Y. Henry, Slow-Wave-Based Nanomagnonic Diode, Phys. Rev. Appl. 14, 024047 (2020).

[26] K. Szulc, P. Graczyk, M. Mruczkiewicz, G. Gubbiotti, and M. Krawczyk, Spin-Wave Diode and Circulator Based on Unidirectional Coupling, Phys. Rev. Appl. 14, 034063 (2020).

[27] Z. Zhang, S. Liu, T. Wen, D. Zhang, L. Jin, Y. Liao, X. Tang, and Z. Zhong, Bias-free reconfigurable magnonic phase shifter based on a spin-current controlled ferromagnetic resonator, J. Phys. D: Appl. Phys. 53, 105002 (2020).

[28] V. V. Kruglyak, C. S. Davies, Y. Au, F. B. Mushenok, G. Hrkac, N. J. Whitehead, S. A. R. Horsley, T. G. Philbin,
V. D. Poimanov, R. Dost, D. A. Allwood, B. J. Inkson, and A. N. Kuchko, Graded magnonic index and spin wave Fano resonances in magnetic structures: Excite, direct, capture, in Spin Wave Confinement. Propagating Waves, edited by S. O. Demokritov (Jenny Stanford Publishing, New York, 2017), Chap. 1.

[29] See Supplemental Material at http://link.aps.org/supplemental/ 10.1103/PhysRevB.104.054437 for the full results of the fitting.

[30] L. D. Landau and E. M. Lifshitz, Electrodynamics of Continuous Media, 2nd ed. (Butterworth-Heinemann, Oxford, 1984).

[31] T. Yu, Y. M. Blanter, and G. E. W. Bauer, Chiral Pumping of Spin Waves, Phys. Rev. Lett. 123, 247202 (2019).

[32] T. Yu and G. E. W. Bauer, Chiral coupling to magnetodipolar radiation, Top. Appl. Phys. 138, 1 (2021).

[33] R. E. Camley, Nonreciprocal surface waves, Surf. Sci. Rep. 7, 103 (1987), and references therein.

[34] R. A. Gallardo, T. Schneider, A. K. Chaurasiya, A. Oelschlägel, S. S. P. K. Arekapudi, A. Roldán-Molina, R. Hübner, K. Lenz, A. Barman, J. Fassbender, J. Lindner, O. Hellwig, and P. Landeros, Reconfigurable Spin-Wave Nonreciprocity Induced by Dipolar Interaction in a Coupled Ferromagnetic Bilayer, Phys. Rev. Appl. 12, 034012 (2019).

[35] V. D. Poimanov, A. N. Kuchko, and V. V. Kruglyak, Scattering of exchange spin waves from a helimagnetic layer sandwiched between two semi-infinite ferromagnetic media, Phys. Rev. B 102, 104414 (2020). 